\title{
A Catalog of Variable Stars Based on the New Name List
}

\author{
E. V. Kazarovets ${ }^{1}$, N. N. Samus' ${ }^{1,2 *}$, O. V. Durlevich ${ }^{2}$, \\ N. N. Kireeva ${ }^{1}$, E. N. Pastukhova ${ }^{1}$, and G. Pojmanski ${ }^{3}$ \\ ${ }^{1}$ Institute of Astronomy, Russian Academy of Sciences, Pyatnitskaya ul. 48, Moscow, 119017 Russia \\ ${ }^{2}$ Sternberg Astronomical Institute, Universitetskii pr. 13, Moscow, 119992 Russia \\ ${ }^{3}$ Astronomical Observatory, University of Warsaw, Al. Ujazdowskie 4, Warsaw, 00-478 Poland
}

Received April 30, 2009; in final form, June 25, 2009

\begin{abstract}
We present a new electronic version of the General Catalog of Variable Stars (GCVS) based on the new IAU name list of confirmed variable stars. The catalog contains 1270 stars, most of them contained earlier in the New Catalog of Suspected Variable Stars or its supplement. A number of recent studiesincluding those by authors of the catalog, who investigated many stars using data from modern automatic surveys, determined light-curve elements for periodic stars, and plotted numerous light curves - have made it possible to move these stars to the GCVS. Among the catalog objects, 24 stars are novae or other unusual variable stars that acquired their GCVS names out of the usual order, upon communication from the Bureau of Astronomical Telegrams of the International Astronomical Union. We present the GCVS names, coordinates, classifications (in two forms: the GCVS system and a new, proposed system), brightnessvariation limits, and light-curve elements for the catalog stars, as well as bibliographic references and remarks when necessary. We discuss several catalog stars that are of astrophysical interest or caused problems during the compilation of the catalog.

PACS numbers: $95.80 .+\mathrm{p}, 97.30 .-\mathrm{b}$

DOI: $10.1134 / \mathrm{S} 1063772909110067$
\end{abstract}

\section{INTRODUCTION}

The General Catalog of Variable Stars (GCVS) is an important international reference source on variable stars in our Galaxy, and is supported by Commissions 27 and 42 (Division $\mathrm{V}$ ) of the International Astronomical Union (IAU). The GCVS contains stars with reliably established variations of their optical brightness. These should be sufficiently well studied that they can be assigned to one of the variablestar types of the GCVS classification system, or can be shown to display unusual brightness variations that do not fit into this classification scheme. Stars are included in the GCVS via the so-called Name Lists of Variable Stars published in the Information Bulletin on Variable Stars (IBVS) of IAU Commissions 27 and 42 . The name lists have been numbered continuously since the 1920s. The GCVS electronic database (http://www.sai.msu.su/gcvs/gevs) provides the latest versions of the catalog, including the name lists; however, in most cases, the information is incomplete for stars of name lists 67-77, which entered the GCVS after the publication of Volumes IIII of its fourth edition [1] in 1985-1987 (only the coordinates, variability types, and variation limits are

*E-mail: samus@sai.msu.ru given for these stars, without light-curve elements or remarks).

We decided to prepare a new version of the GCVS for listed stars to accompany future publications of name lists, expected to occur every two to three years, so that we can expediently add information on stars to be added to the GCVS database in full GCVS format.

\section{THE CATALOG}

The 79th Name List of Variable Stars [2] contains 1270 stars, which obtained their final names in the GCVS system in 2007-2008. After the publication of this name list, the number of genuine stars with names in the GCVS system reached 41483 . This does not include named "stars" that actually do not exist, such as defects in photographic plates or minor planets, but does include stars whose variability was doubted after their naming, so that they were marked as probably constant ("types" cst or cst:) in the cata$\log$. A characteristic feature of the 79th Name List is that its stars were selected almost exclusively among stars that had earlier entered the New Catalog of Suspected Variable Stars (the NSV catalog [3]) or its supplement [4]. Catalogs of suspected variable stars include poorly studied stars for which the character 
of any variations remains unclear (and sometimes with the very presence of variations being doubtful). The NSV catalog [3] replaced two catalogs of suspected variable stars published earlier by the GCVS team $[5,6]$.

The tables of the name list are available at http:// www.sai.msu.su/gevs/gevs/nl79/ and at the IBVS web site. They contain the coordinates, brightnessvariations limits, variability types, and identifications with other catalogs.

Stars of the NSV catalog are usually transferred to the GCVS after the publication of sufficiently detailed studies. Recent studies have opened unprecedented possibilities for moving numerous stars from catalogs of suspected variables to the GCVS, based on information obtained from large-scale automated sky surveys, and most importantly, from the ASAS-3 [7] and ROTSE-I/NSVS [8] surveys. We are systematically studying objects in the NSV catalog in order to obtain possible identifications, derive accurate coordinates, and search for information (primarily from automated sky surveys) sufficient to confirm variations and determine the variability type and light-curve elements.

In addition to stars from the NSV catalog, the 79th Name List contains 24 stars, novae, or variables of other rare types whose GCVS names were first announced in IAU Circulars. The GCVS team names such stars quickly, upon communication from the IAU Bureau of Astronomical Telegrams.

The electronic catalog in the GCVS system compiled during the preparation of the 79th Name List of Variable Stars consists of a file in the format of the main part of the GCVS (with several additional columns), a file of detailed position information, a file of remarks, and a file of bibliographic references with bibliographic codes in the ADS style. This catalog structure is intended to achieve maximum format compatibility with the files of the GCVS (4th edition) Volumes I-III provided in our database.

The file http://www.sai.msu.su/gevs/gevs/nl79/ nl79_5ed/nl79_5ed.dat presents the main part of the catalog, compiled using the traditional GCVS variable-star classification scheme (http://www.sai. msu.su/gcvs/gcvs/iii/vartype.txt), as well as the experimental variable-star classification scheme we proposed to the IAU, described at http://www.sai. msu.su/gcvs/future/classif.htm. Its columns contain the following information.

1. Six-digit number of the variable star in the internal numbering system of the GCVS (the first two digits are the ordinal number of the constellation, and the last four digits the variable star's number in the constellation).

\section{GCVS name.}

3. Equatorial coordinates $\mathbf{J} 2000.0$, rounded to $0.1^{\mathrm{s}}$ in right ascension and $1^{\prime \prime}$ in declination.

4. Variability type in the new classification scheme, described at http://www.sai.msu.su/gevs/ future/classif.htm.

5. Variability type in the GCVS classification scheme (http://www.sai.msu.su/gevs/gevs/iii/ vartype.txt).

6-9. Variability range and photometric band of the magnitudes. The first magnitude column gives the magnitude at maximum brightness, the second the magnitude at minimum brightness, and the third the magnitude at the secondary minimum, for eclipsing variables. The symbols $U, B, V, R$ in this column specify the center of the photometric band in the Johnson $U B V R I$ system; Hp are magnitudes in the Hipparcos system [9]; p are photographic magnitudes in old systems; $J$ and $K s$ are magnitudes in the corresponding infrared bands $J$ and $K_{s}$. The symbol "*” stands for instrumental CCD magnitudes, which are, as a rule, relative to red magnitudes of comparison stars. Instead of the magnitude at minimum, the corresponding column may contain the amplitude of brightness variations in brackets. This means that a provisional magnitude (preferably at maximum, but possibly at a different phase, or the mean magnitude) is given instead of the magnitude at maximum. If the amplitude is given for a photometric band different from that of the quoted magnitude, this band is also indicated in brackets. Note that the GCVS traditionally presents the widest reliably observed variation ranges, and the amplitude is understood as the magnitude difference between minimum and maximum (rather than the mean amplitude or the amplitude of the periodic signal).

10-12. Light-curve elements, i.e., epoch and period. The first of these columns presents epochs as Julian dates minus 2400000 . Epochs of brightness maxima are given for most pulsating variables; epochs of minima are given for RV stars and eclipsing variables. The next column, used only for novae, presents the year of the observed brightness maximum. Periods in days follow. Note that, in many cases of RRAB stars whose periods were taken from the ASAS-3 catalog [7], our catalog provides significantly corrected epochs of maxima, since the software used in the ASAS-3 project is not very successful in reducing light curves that differ substantially from harmonic functions.

13. $M-m$ or $D$ are the traditional lightcurve parameters. For pulsating variables, the cata$\log$ gives $(M-m)$, or the fraction of the entire period 
occupied by the ascending branch; the parameter presented for Algol-type eclipsing stars is $D$, or the duration of the primary eclipse as a fraction of the entire period. Both parameters are expressed in hundredths of the period.

14. The star's spectral type, if known from any sources. As a rule, we do not reproduce spectral types derived from photometry in the catalog.

15. Two bibliographic references. As is usual in the GCVS, the first reference is to a study of the star (as a rule, the source of the catalog period or, in the absence of a period, of the variability type), while the second is to a publication containing a finding chart. Instead of a findingchart reference, the second of these columns may present a catalog containing the star's coordinates and magnitude, enabling identification (USNO is a catalog of the US Naval Observatory; A2.0 or B1.0 are $[10,11]$; GSC the Hubble Space Telescope Guide Star Catalog, version 1.2 [12]; DM the "Durchmusterung" catalogs, Bonner Durchmusterung, Cordoba Durchmusterung, Cape Photographic Durchmusterung; and 2MASS the 2MASS Point Source Catalog [13]). Other bibliographic references in both subcolumns are numbers corresponding to the list of references in the file http:// www.sai.msu.su/gevs/gevs/nl79/nl79_ref.txt.

Information on the coordinates of the catalog variable stars is presented in a more accurate (to $0.01^{\mathrm{s}}$ in right ascension and $0.1^{\prime \prime}$ in declination) and detailed form in the file http://www.sai.msu.su/gevs/gcvs/ nl79/nl79_5ed/nl79_pos.dat. The columns of this file give the internal G $\overline{C V S}$ number of the star and its GCVS name, followed by the accurate coordinates and proper motion (J2000.0) of the star and a reference to a positional catalog or source of positional information. If the star's proper motion is given, this means that the coordinates were reduced from the epoch of the positional catalog to epoch 2000.0. If the proper motion is unknown, the coordinates are for the equinox J2000.0 and the indicated epoch. We used the following sources for the coordinates.

2MASS: The Two Micron All Sky Survey [13].

A2.0: The Catalog of Astrometric Standards [10].

AC: The Catalog of Four Million Stars (cf. [14]).

B1.0: The All-Sky USNO B1.0 Catalog of 1045913669 Sources [11]. [15].

GSC2.3: The Guide Star Catalog, Version 2.3

Hip: The Hipparcos Catalog [9].

Lit.: coordinates from various sources in the literature.

Npm: The Lick Northern Proper Motion Program [16].

ASTRONOMY REPORTS Vol.53 No. 112009
Tyc2: The Tycho-2 Catalog [17].

UCAC2: US Naval Observatory CCD Astrograph Catalog [18].

Byte-by-byte summaries of the above files can be found at http://www.sai.msu.su/gevs/gevs/nl79/ nl79_5ed/ReadMe.htm.

The file ttp://www.sai.msu.su/gevs/gevs/nl79/ nl79_5ed/nl79_rem.txt presents text remarks. These may contain information on the presence of visual companions, asymmetry of the secondary relative to the primary minimum, possible alternative periods, descriptions of the character of brightness variations for unusual variables, and other information. The presence of a remark is indicated with an asterisk after the star's GCVS name in the main table.

\section{STATISTICS ON VARIABILITY TYPES IN THE CATALOG}

Because of the character of variable-star selection for the 79th Name List (almost exclusively via catalogs of suspected variable stars), the statistics of variability types in the new catalog is considerably different from that for the GCVS in general. For example, Mira stars, which have large variability amplitudes, were left in the suspected-variable list somewhat less often than is typical. Whereas Mira stars comprise almost $19 \%$ of the 40368 variables in Volumes I-III of the 4th GCVS, supplemented with the 67th-78th Name Lists, our new catalog has only 150 Miras ( $12 \%$ of the total number of stars). There are even fewer RR Lyrae variables in the new catalog: 108, or less than $9 \%$ of the total (in the earlier combined statistics, about $18 \%$ of the catalog stars were RR Lyraes). The variables represented most widely are eclipsing binaries of all kinds ( 499 objects, or $39 \%$ ), whose fraction in the combined statistics is only $16.5 \%$ : it is precisely eclipsing stars, especially Algol variables, that often remain the category of suspected variables for the longest times. Four hundred stars $(31 \%)$ are red sem-iregular and irregular pulsating variables of all subtypes (about $24 \%$ in the combined statistics). As expected, Cepheids are rare (a total of 35 Population I and II Cepheids, or $3 \%$, with the fraction in the combined statistics being not very different, $2 \%$ ). The special ordering for adding novae to the 79th Name List led to an excess of such objects (20, or more than $1.5 \%$ ) compared to the combined GCVS statistics (0.7\%).

Other types of variable stars are more poorly represented in the catalog. Note, however, that, somewhat unexpectedly, the catalog also contains rare types of variable stars (two probable symbiotic stars; five high-amplitude $\delta$ Scuti stars and one SX Phoenicis star; four RV Tauri stars; seven dwarf novae and two 


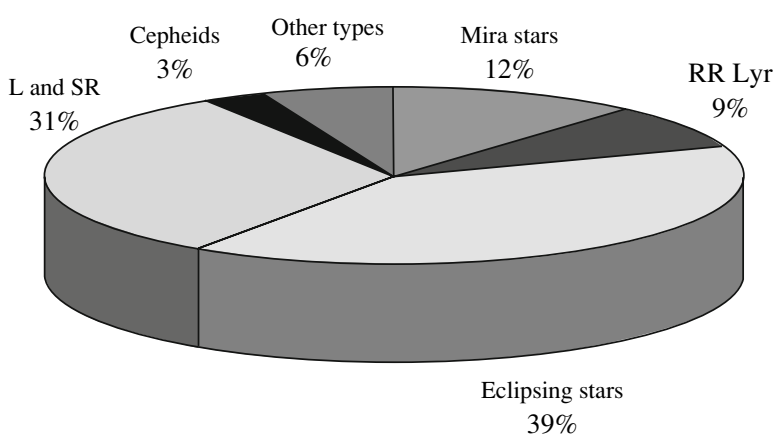

Fig. 1. The statistics of variability types in the catalog compiled for the 79th Name List of Variable Stars.

nova-like stars, one of them also displaying pulsations of the white dwarf; one $\mathrm{X}$-ray nova, etc.). Two stars (see Section 4) do not quite fit the existing classification systems.

Figure 1 shows the statistics of variable-star types in our new catalog.

\section{SOME OBJECTS \\ FROM THE 79th NAME LIST}

In this section, we discuss several interesting or problematic variable stars in the electronic variablestar catalog of the 79th Name List.

V1710 Aql. This star has unusual characteristics not admitting a straightforward description in the existing classification system ("type $*$ "). The nucleus of the planetary nebula PK 36-1 ${ }^{\circ} 1$ (spectral type B from direct spectroscopic data) reveals highamplitude variations (up to $1^{m}$ in the $V$ band) with a period that is unusually long for hot stars. Its period, $68.06^{\mathrm{d}}$, has apparently remained constant for many decades [19].

V613 Car. This Algol is not contained in the ASAS-3 catalog of variable stars [7]. Data from the ASAS-3 survey were used by Otero et al. [20] to study the star. However, the table in their paper gives a period that is twice the actual period $(P=$ $\left.11.8884^{\mathrm{d}}\right)$, though the light curve presented in the same paper is plotted with the correct period, $5.9442^{\mathrm{d}}$. The correctness of the shorter period is supported by obvious traces of the secondary minimum. Note that the ASAS-3 observations, obtained with a short focal length, do not separate the stars GSC 8941.00668 and GSC 8941.00913, separated by an angular distance of about $15^{\prime \prime}$. The identification of V613 Car with the first of these two GSC stars, accepted by us as well as by Otero et al. [20], is based on the photographic magnitude at maximum, $12.5^{\mathrm{m}}$, published in the discovery paper [21]; the second GSC star is much fainter. At the same time, the amplitude claimed by Luyten $[21]\left(3^{m}\right)$ is too high, even if we take into account that the amplitude in the ASAS-3 survey is suppressed by the presence of a close companion.

V1129 Cen. This EB eclipsing variable with a period of $0.89^{\mathrm{d}}$ brightens by several tenths of a magnitude in the $V$ band once each 357 days; each brightening is observable for about a month (S. Otero, http://ar.geocities.com/varsao/NSV_19448.htm). The simplest explanation-the presence of a Miratype visual companion-can be rejected due to the absence of any considerable infrared excess. Walter et al. [22] found HeII emission during a brightening, and suggested the presence of a compact object on an eccentric orbit in the system. The type given in the Name List is EB+*.

MX Dra. Hoogeveen [23] considered the star to be a Population II Cepheid, though he noted its possible association with an X-ray source. In our opinion, there is no doubt that the object is associated with the X-ray source 1RXS J121215.7+685306. This is a non-eclipsing RS CVn star, like many other "Cepheids" in the NSVS survey. This classification can be directly suggested based on Hoogeveen's light curve, which shows an inverse asymmetry (the descending branch is steeper than the ascending branch), which varies from cycle to cycle.

V461 Hya. The variable was discovered at Harvard Observatory; no finding chart was published. In the course of our work on refining the coordinates of variable stars [24-26], this star was found, together with thousands of other Harvard variables, by Martha Hazen, using logbooks and sketches kept in the USA. It now turns out that Hazen's identification for V461 Hya is wrong. The star was confidently detected in the ASAS-3 data (but is not present in the ASAS-3 variable-star catalog). It is probably a shortperiod eclipsing variable.

PX Mus. In the name list [2], this star is assigned to the rare type RVB, with its classification 


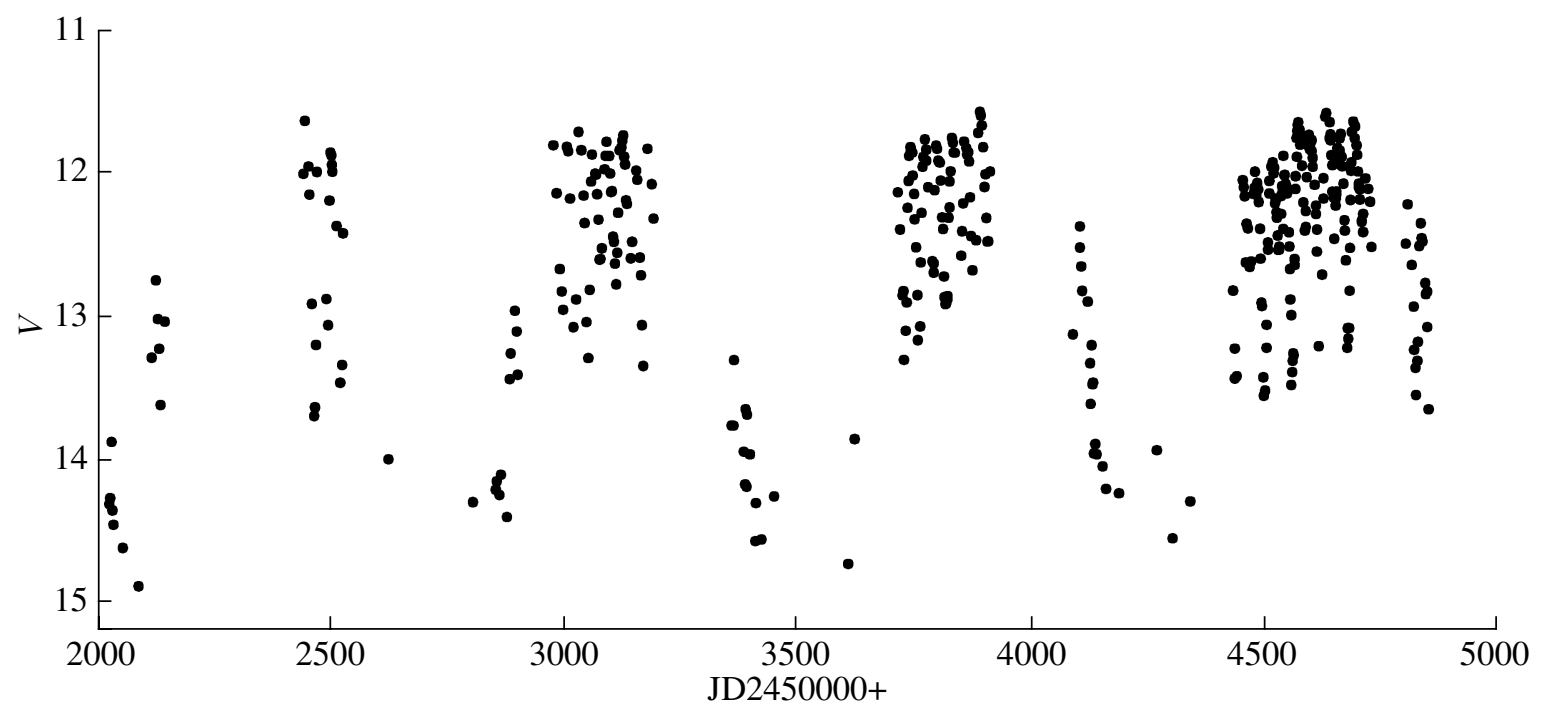

Fig. 2. Light curve of the variable PX Mus (type RVB).

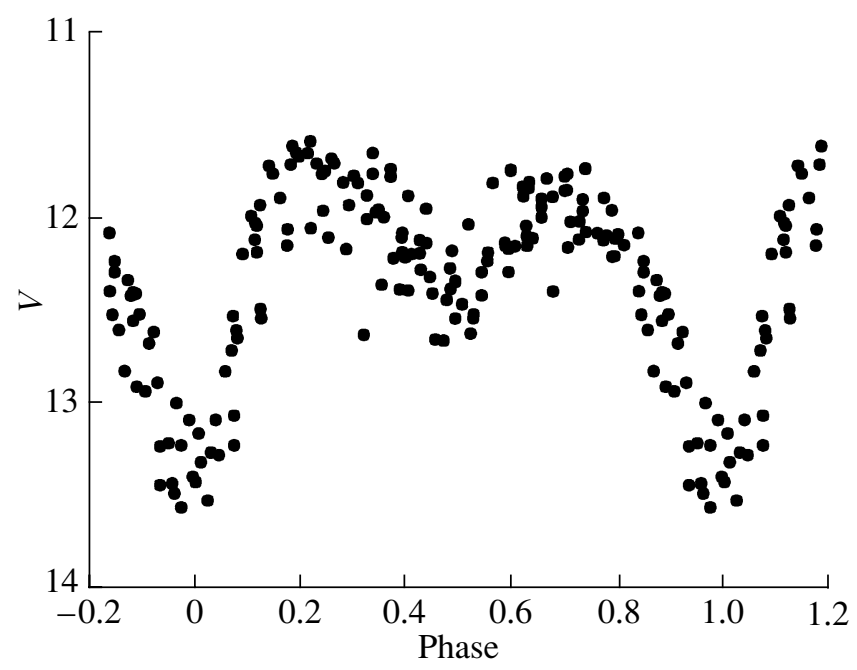

Fig. 3. Phased light curve of PX Mus for JD 2454430-2454730 plotted with the period 59.6 ${ }^{\mathrm{d}}$.

marked uncertain. The star is not in the variablestar catalog of the ASAS-3 survey [7], but the survey's photometric data can be used to study the variable, and even refine the name-list information. As of January 23, 2009, the ASAS-3 photometric catalog contained $401 \mathrm{~V}$-band observations of this star, not counting those with the worst accuracy (flag D in the photometric catalog) and estimates when the star was actually not detected (flag C). The observations cover the time between JD 2451913 and JD 2454851. These data suggest that the star is a certain RVB variable, with a total range of brightness variations from 11.6 to $14.7 V$ (Fig. 2). Four cycles of the long-period variations can be followed $\left(P \approx 770^{\mathrm{d}}\right)$. At the maximum brightness of the long-period variations, alternating minimum depths are clearly ob- served, with the period between the primary minima being about 60 days (Fig. 3) and variations approximately between 11.6 and $13.2 \mathrm{~V}$.

V366 Sge. This star is an EB eclipsing variable. In the ASAS-3 catalog, it is identified, though uncertainly, with the Mira NN Sge. This identification is erroneous. The eclipsing star's coordinates are $19^{\mathrm{h}} 18^{\mathrm{m}} 13.8^{\mathrm{s}},+17^{\circ} 39^{\prime} 14^{\prime \prime}(\mathrm{J} 2000.0)$, while the correct coordinates of NN Sge are $19^{\mathrm{h}} 18^{\mathrm{m}} 03.1^{\mathrm{s}},+17^{\circ} 41^{\prime} 47^{\prime \prime}$ (J2000.0). The true NN Sge was not detected in the ASAS-3 survey, but variations can be noted when comparing the POSSI and POSSII red images of the US Naval Observatory Image and Catalog Server (http://www.nofs.navy.mil/data/FchPix/). Another very red star, does not show any variations 


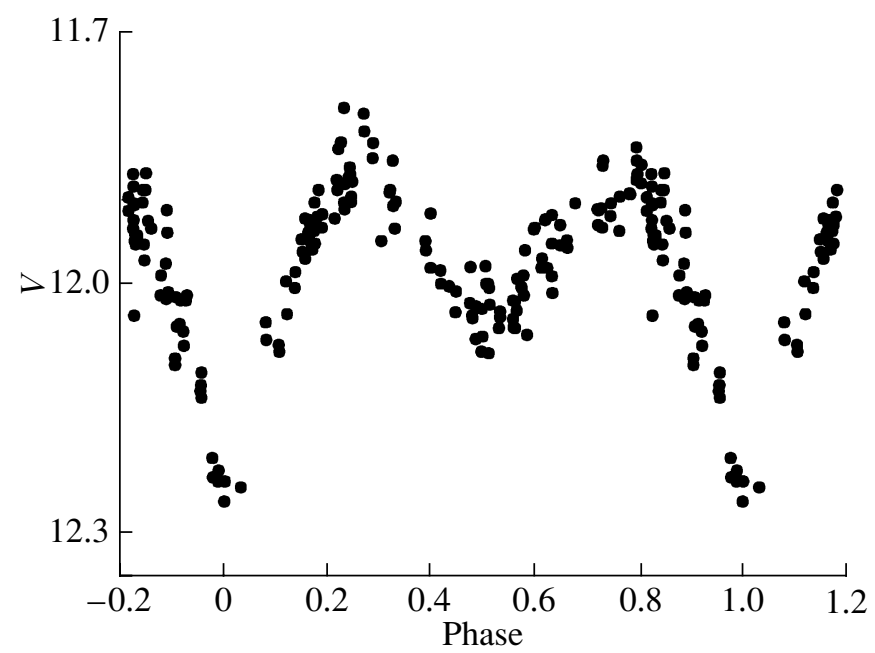

Fig. 4. Phased light curve of V366 Sge (type EB) for the elements MinI $=2451456.676+0.751779^{\mathrm{d}} E$.

in the NSVS survey, is present at $19^{\mathrm{h}} 18^{\mathrm{m}} 05.5^{\mathrm{s}}$, $+17^{\circ} 41^{\prime} 51^{\prime \prime}$ (J2000.0). Our light-curve elements of V366 Sge were derived from the combined ASAS-3 and NSVS data, and are improved compared to those of Khruslov [27], which were based solely on NSVS observations. The star exhibits the O'Connell effect (maxima of different height). Its NSVS light curve with our elements is shown in Fig. 4.

V420 Vel. The problem of this star is related to its identification with the "Durchmusterung" catalogs. Strohmeier et al. [28] announced their detection, on photographic plates, of a variable star BV $700=$ NSV 4781, identified by them with CoD $-45^{\circ} 5845$. Their paper contains finding charts for several stars, but, according to an old tradition, not for "Durchmusterung" stars. Within one minute of arc from $\operatorname{CoD} 45^{\circ} 5845=\mathrm{CPD} 45^{\circ} 4455$ is another star, CoD $45^{\circ} 5842$, that is missing from the CPD catalog. While $\mathrm{CoD} 45^{\circ} 5845$ shows no considerable variations in the ASAS- 3 data, $\mathrm{CoD} 45^{\circ} 5842$ enters the ASAS-3 catalog of variable stars as a periodic variable $\left(P=0.60508^{\mathrm{d}}\right)$. The star's type from the ASAS-3 catalog, DCEP-FO, should be changed to $\mathrm{RRAB}$, as the light curve is obviously asymmetric. We believe that the Strohmeier et al. [28] erroneously assigned the variable the number of a neighboring $\mathrm{CoD}$ star and, as was done in the ASAS-3 catalog, we identify V420 Vel, an RR Lyrae star in our new catalog, with NSV 4781.

\section{CONCLUSIONS}

We have presented a catalog in the full GCVS format for the just published Name List of Variable Stars. The catalog contains 1270 stars from the 79th Name List [2]. Almost all of these stars were earlier contained in the NSV catalog or its supplement. Our new catalog presents coordinates, two versions of the classification, variability limits, light-curve elements for periodic stars, remarks, and bibliographic references. It is openly accessible to users at the GCVS web site. We have considered in more detail several stars that caused problems in compiling the catalog or were found to be of special astrophysical interest for various reasons.

\section{ACKNOWLEDGMENTS}

This work was supported by the Russian Foundation for Basic Research (project code 08-02-00375), the Program of State Support to Leading Scientific Schools of the Russian Federation, and the Basic Research Program of the Presidium of the Russian Academy of Sciences "The Origin, Structure, and Evolution of Objects in the Universe." We thank S.V. Antipin for his assistance during the preparation of the manuscript.

\section{REFERENCES}

1. P. N. Kholopov, N. N. Samus', M. S. Frolov, et al., General Catalog of Variable Stars, 4th ed., vols. 13 (Nauka, Moscow, 1985-1987) [in Russian].

2. E. V. Kazarovets, N. N. Samus, O. V. Durlevich, et al., Inform. Bull. Var. Stars № 5863 (2008).

3. B. V. Kukarkin, P. N. Kholopov, N. M. Artyukhina, et al., New Catalog of Suspected Variable Stars (Nauka, Moscow, 1982) [in Russian].

4. E. V. Kazarovets, N. N. Samus, and O. V. Durlevich, Inf. Bull. Var. Stars № 4655 (1998).

5. B. V. Kukarkin, P. P. Parenago, Yu. I. Efremov, and P. N. Kholopov, Catalog of Suspected Variable Stars (Akad. Nauk SSSR, Moscow, 1951) [in Russian]. 
6. B. V. Kukarkin, P. N. Kholopov, Yu. N. Efremov, and N. E. Kurochkin, Second Catalog of Suspected Variable Stars (Astrosovet AN SSSR, GAISh, Moscow, 1965) [in Russian].

7. G. Pojmanski, Acta Astron. 52, 397 (2002).

8. P. R. Woźniak, W. T. Vestrand, C. W. Akerlof, et al., Astron. J. 127, 2436 (2004).

9. The Hipparcos and Tycho Catalogs, Eur. Space Agency Publ., SP-1200 (ESA, 1997).

10. D. Monet, A. Bird, B. Canzian, et al., USNO-A V2. 0. A Catalog of Astrometric Standards, $11 \mathrm{CD}$ ROMs (US Naval Observatory, Washington, 1998).

11. D. G. Monet, S. E. Levine, B. Casian, et al., Astron. J. 125, 984 (2003).

12. J. E. Morrison, S. Röser, B. McLean, et al., Astron. J. 121, $1752(2001)$.

13. R. M. Cutri, M. F. Skrutskie, S. Van Dyk, et al., The 2MASS All-Sky Catalog of Point Sources, Centre de Données Astronomiques de Strasbourg, II/246 (2003).

14. The Four-Million Star Catalog, Ed. by A. P. Gulyaev and V. V. Nesterov (Mosc. Gos. Univ., Moscow, 1992) [in Russian].

15. B. Lasker, M. G. Lattanzi, B. J. McLean, et al., Astron. J. 136, 735 (2008).

16. A. R. Klemola, R. B. Hanson, and B. F. Jones, The Lick Northern Proper Motion Program, Centre de Données Astronomiques de Strasbourg, I/199 (1987).
17. E. Høg, C. Fabricius, V. V. Makarov, et al., The Tycho2 Catalog, CD-ROM (Copenhagen, 2000).

18. N. Zacharias, S. E. Urban, M. I. Zacharias, et al., Astron. J. 127, 3043 (2004).

19. J. Jurcsik, in IAU Symp. 155: Planetary Nebulae, Ed. by R. Weinberger and A. Acker (Kluwer Acad., Dordrecht, 1993), p. 399.

20. S. A. Otero, P. Wils, and P. A. Dubovsky, Inform. Bull. Var. Stars № 5586 (2005).

21. W. J. Luyten, Astron. Nachr. 246, 437 (1932).

22. F. Walter, H. E. Bond, and A. Pasten, IAU Circ. № 8663 (2006).

23. G. J. Hoogeveen, Inform. Bull. Var. Stars № 5652 (2005).

24. N. N. Samus', V. P. Goranskiu1, O. V. Durlevich, et al., Pis'ma Astron. Zh. 28, 201 (2002) [Astron. Lett. 28, $174(2002)]$.

25. N. N. Samus', V. P. Goranskiu1, O. V. Durlevich, et al., Pis'ma Astron. Zh. 29, 532 (2003) [Astron. Lett. 29, 468 (2003)].

26. N. N. Samus', O. V. Durlevich, A. V. Zharova, et al., Pis'ma Astron. Zh. 32, 295 (2006) [Astron. Lett. 32, 263 (2006)].

27. A. V. Khruslov, Inform. Bull. Var. Stars № 5699, 12 (2006).

28. W. Strohmeier, R. Knigge, and H. Ott, Inform. Bull. Var. Stars № 107 (1965).

Translated by N. Samus' 